\title{
How many bits should be reported in quantized cooperative spectrum sensing?
}

\author{
Nhan Nguyen-Thanh, Philippe Ciblat, Sina Maleki and Van-Tam Nguyen
}

\begin{abstract}
We introduce an algorithm for optimizing sensing parameters including the number of sensing samples and the number of reporting bits of a quantization-based cooperative spectrum sensing scheme in cognitive radio networks. This is obtained by maximizing the network throughput subject to a target detection probability. With Rayleigh fading and energy detector, the proposed algorithm simultaneously optimizes the number of sensing samples at a local node, the number of bits for quantizing local sensing data and the global threshold at a fusion center.
\end{abstract}

Index Terms-cognitive radio, spectrum sensing, cooperative, multibit decision, quantization, sensing-throughput tradeoff.

\section{INTRODUCTION}

Cognitive radio (CR), which enables secondary access to licensed bands, is a promising candidate for enhancing the utilization of the scarce spectrum resource in future communication systems. A secondary user can be permitted to use licensed spectrum, provided that it does not interfere with any primary users. This means that $\mathrm{CR}$ should be able to exploit spectrum holes by detecting them and using them in a cognitive manner. A widespread approach for characterizing the spectrum usage of primary systems is the so-called spectrum sensing [1], [2].

Spectrum sensing at terminals may not provide sensing results as accurate as required because of deep shadowing or fading. To deal with this problem, a fusion center (FC) collects sensing information from multiple terminals to eventually obtain a more reliable decision. This method is called cooperative spectrum sensing [3]-[5]. Main works related to cooperative sensing dealt with the design of local sensing algorithm, the combination of the local parameters at the FC (see [1] and references therein). In contrast, only a few works have been devoted to the optimization of the whole secondary system, especially by finding the trade-off between the duration of the sensing step and that of the data transmission step [6]-[8]. An efficient way to exhibit this trade-off is to maximize the throughput [6] with respect to the sensing duration with 1bit hard decision and conventional fusion rules. However, the duration for reporting local information from each CR to the FC, which is linearly related to the quantizer resolution of the local decision, has never been optimized. The reporting step for 1-bit hard decision and even soft decision has been taken into account only through sensing performance [3], [8][10]. Obviously, if the reporting time is too short and so carries a degraded version of the local information (in the worst case, 1 bit), the sensing decision at the FC may not be reliable and the whole system may not perform well. In contrast, if the reporting time is too long, the time devoted to data transmission may be too short and the required data rate may not be fulfilled. As a consequence, this paper deals with the number of bits allowed for quantizing local sensing information. This means that our work has a strong connection to the problem of selecting hard decision or soft decision in [5], [8].

The trade-off between the sensing process length and the utilization channel time is investigated by formulating an optimization problem of maximizing the network throughput under the constraint of primary system protection requirement. The algorithm to find the optimal number of sensing samples and the optimal number of reporting bits is proposed.

\section{SYSTEM MODEL}

We consider a CR network with $K$ users. The CR network utilizes opportunistic spectrum access for sharing spectrum bands with primary systems. Cooperative sensing is adopted to detect primary users. The cooperative sensing scheme includes two steps. The first step consists of spectrum sensing of CR users. The second step is sensing result reporting to the FC, which makes a final decision on the primary user state.

In this work, we consider the energy detection method for the first step because of its simple implementation and its robustness to unknown information of the source signal and channel fading [11], [12]. For the second step, the reporting is done through a control channel with a fixed limited bandwidth [9], [13]. Since every methods of orthogonal multiple access, e.g., Time Division Multiple Access (TDMA), Frequency Division Multiple Access, etc., offer the same spectral efficiency, they can be used equivalently. For the sake of simplicity of the presentation and without lost of generality, we consider TDMA scheme and a soft data fusion rule with multi-bit local decisions at the FC. The structure of the operation frame is illustrated in Fig. 1.

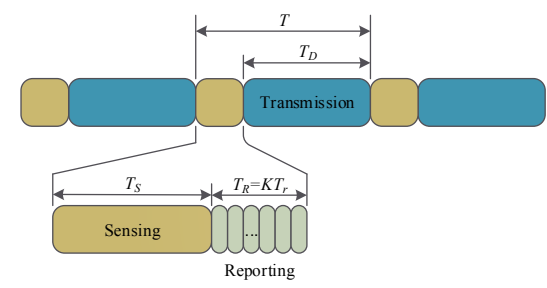

Fig. 1. Frame structure of the CR network.

The local spectrum sensing is a binary hypothesis testing problem as follows

$$
y_{k}[n]=\left\{\begin{array}{lll}
w_{k}[n], & H_{0} & n=1,2, \ldots, N \\
h_{k} s[n]+w_{k}[n], & H_{1} & k=1,2, \cdots, K
\end{array}\right.
$$


where $y_{k}[n]$ is the received signal at time $n$ at the $k$-th CR user, $w_{k}[n]$ is the added noise and is assumed to be a zero-mean i.i.d. complex-valued circularly-symmetric Gaussian process with variance $\sigma_{w_{k}}^{2}$ per complex dimension, $s[n]$ is the potential unknown deterministic signal coming from the primary user, and $h_{k}$ is the block-fading channel gain between the primary user and the $k$-th CR user. $N$ is the number of sensing samples, ( $N=f_{S} T_{S}$, where $f_{S}$ is the sampling frequency and $T_{S}$ is the sensing time). $H_{0}$ and $H_{1}$ represent the hypotheses of the absence and the presence of primary signal, respectively. We consider that the channel is a slow Rayleigh flat fading with variance $\sigma_{h_{k}}^{2}$. The channel realization is generated independently frame by frame as done in [11], [12].

The test statistic of the energy detector is given by $z_{k}=$ $\sum_{n=1}^{N}\left|y_{k}[n]\right|^{2}$. Given $h_{k}$, it has been shown in [14] that $z_{k}$ has central and non-central chi-squared distribution under $H_{0}$ and $H_{1}$, respectively. The test statistic can be then described by

$$
z_{k} \sim\left\{\begin{array}{cc}
\chi_{2 N}^{2}, & H_{0} \\
\chi_{2 N}^{2}\left(2 N \gamma_{k}\right), & H_{1}
\end{array}\right.
$$

where $\gamma_{k}=\left|h_{k}\right|^{2} E_{s} / \sigma_{w_{k}}^{2}$ is the instantaneous Signal-to-Noise Ratio (SNR) of the received signal at the $k$-th user with the symbol variance $E_{s}$. Given $h_{k}$, the cumulative density functions (cdf) of the test are thus computed by

$$
\begin{aligned}
F_{z_{k} \mid H_{0}}\left(z \mid H_{0}\right) & =P_{N}(z / 2) \\
F_{z_{k} \mid H_{1}, h_{k}}\left(z \mid H_{1}, h_{k}\right) & =1-Q_{N}\left(\sqrt{2 N \gamma_{k}}, \sqrt{z}\right)
\end{aligned}
$$

where $Q_{N}(.,$.$) denotes the generalized Marcum Q-function,$ $P_{N}(b)=\gamma(N, b) / \Gamma(N)$ with the gamma function $\Gamma($.$) and$ the incomplete gamma function $\gamma(.,$.$) .$

As $h_{k}$ is a Rayleigh channel, the SNR $\gamma_{k}$ follows an exponential probability density function (pdf) given by $f\left(\gamma_{k}\right)=1 / \bar{\gamma}_{k} \exp \left(-\gamma_{k} / \bar{\gamma}_{k}\right)$, where $\bar{\gamma}_{k}=\sigma_{h_{k}}^{2} E_{s} / \sigma_{w_{k}}^{2}$ is the average SNR received at the $k$-th user. Using Eq. (9) in [12] and Section 8.35 in [15], we obtain the cdf and the pdf of $z_{k}$ under $H_{1}$ as follows.

$$
\begin{aligned}
F_{z_{k} \mid H_{1}}\left(z \mid H_{1}\right) & =P_{\nu}\left(\frac{z}{2}\right)-e^{\frac{-z}{2 M_{k} N \bar{\gamma}_{k}}} M_{k}^{\nu} P_{\nu}\left(\frac{z}{2 M_{k}}\right) \\
f_{z_{k} \mid H_{1}}\left(z \mid H_{1}\right) & =\frac{e^{\frac{-z}{2 M_{k} N \bar{\gamma}_{k}}}}{2 M_{k} N \bar{\gamma}_{k}} M_{k}{ }^{\nu} P_{\nu}\left(\frac{z}{2 M_{k}}\right)
\end{aligned}
$$

where $M_{k}=1+1 /\left(N \bar{\gamma}_{k}\right)$ and $\nu=N-1$.

The cdf of $z_{k}$ under $H_{0}$ is the same as that of Eq. (2). Since it is independent of the fading, its pdf is given by

$$
f_{z_{k} \mid H_{0}}\left(z \mid H_{0}\right)=\frac{z^{N-1} e^{-\frac{z}{2}}}{2^{N} \Gamma(N)} .
$$

After the sensing period, each energy test is reported to the FC, where a squared-law combining is adopted [12], and the global test is then given by

$$
Z=\sum_{k=1}^{K} z_{k} \underset{H_{0}}{\stackrel{H_{1}}{\gtrless}} \eta
$$

where $\eta$ is the decision threshold.

\section{QUANTIZED COOPERATIVE SENSING}

Reporting a raw $z_{k}$ requires time, bandwidth and energy. It is therefore relevant to communicate with the FC through a quantized version of the test statistic, which corresponds to work with a multi-bit decision at the local nodes. The realvalued (also called raw or soft) energy $z_{k}$ is replaced with its $B$-bit quantized version in Eq. (7). The practical test at the FC then becomes

$$
Z^{(B)}=\sum_{k=1}^{K} z_{k}^{(B)} \underset{H_{0}}{\stackrel{H_{1}}{\gtrless}} \eta^{(B)}
$$

where $z_{k}^{(B)}=Q_{k}^{(B)}\left(z_{k}\right)$ is the quantized version of $z_{k}$ and $Q_{k}^{(B)}$ denotes a $B$-bit quantizer associated with the $k$-th user. Let $M$ the number of quantization levels, then $M=2^{B}$. Let $\left\{t_{k, i}\right\}_{i=0}^{M}$ and $\left\{L_{k, j}\right\}_{j=1}^{M}$ the set of thresholds and the set of quantization levels for $Q_{k}^{(B)}$, respectively. As the support of the pdf of $z_{k}$ is $\mathbb{R}_{+}$, we have $t_{k, 0}=0, t_{k, M}=+\infty$, and $\Re_{k, i}=$ $\left[t_{k, i-1}, t_{k, i}\right), i=1, \ldots, M . \Re_{k, i}$ denotes the $i$-th quantization region of the $k$-th user. The quantization level is usually the central point of the quantization region. Hence, we have

$$
L_{k, i}=\frac{1}{S_{k, i}} \int_{\Re_{k, i}} z f_{z_{k}}(z) d z
$$

where $S_{k, i}=\int_{\Re_{k, i}} f_{z_{k}}(z) d z$ and $f_{z_{k}}=\pi_{0} f_{z_{k} \mid H_{0}}+(1-$ $\left.\pi_{0}\right) f_{z_{k} \mid H_{1}}$ with $\pi_{0}$ the probability of primary user inactivity.

The following quantizers are hereafter considered:

- Uniform quantizer: The quantization thresholds are given by $t_{k, i}=t_{k, i-1}+\Delta_{k}, i=1, \ldots, M-1$, where $\Delta_{k}=t_{k, \max } / M . t_{k, \max }$ is an artificial threshold for defining a maximum support of $z_{k}$. Here, it is selected such that $\int_{0}^{t_{k, \max }} f_{z_{k}}(z) d z=1-10^{-6}$.

- Minimum mean square error (MMSE) quantizer [16]: This quantizer aims at minimizing the quantization error. The levels and thresholds (with $t_{k, i}=\left(L_{k, i}+L_{k, i+1}\right) / 2$ ) can be found by using Lloyd-Max algorithm.

- Maximum entropy (ME) quantizer [17]: The quantization thresholds $t_{k, i}$ are obtained by forcing $S_{k, i}=1 / M, \forall i=1, \ldots, M$.

In order to perform the quantization and the dequantization, the local user $k$ needs its quantization thresholds, and the FC needs the pdf of $z_{k}$. If the coherence time of the statistics of $z_{k}$ is large enough, the report of the pdf from the user to the FC will be rarely performed. When the report can not be implemented or when the statistics of $z_{k}$ can not be archived, $z_{k}$ can be considered as a uniformly distributed process, and so the uniform quantizer with $L_{i}=(i-1 / 2) \Delta$ (where $\Delta$ is a pre-defined term independent of the user) is well adapted.

To determine the threshold $\eta^{(B)}$, the probability mass function (pmf) of $Z^{B}$ under $H_{0}$ and $H_{1}$ is needed. Since the test at the FC, given by Eq. (8), is the sum of the $K$ local independent tests, its pdf, denoted by $f_{Z^{(B)} \mid H_{j}}$, is obtained by

$$
f_{Z^{(B)} \mid H_{j}}=f_{z_{1}^{(B)} \mid H_{j}} \star f_{z_{2}^{(B)} \mid H_{j}} \star \cdots \star f_{z_{K}^{(B)} \mid H_{j}}
$$

where $\star$ denotes the convolution operator, and $f_{z_{k}^{(B)} \mid H_{j}}$ is the pmf of $z_{k}^{(B)}$ under $H_{j}$ and is given by

$$
f_{z_{k}^{(B)} \mid H_{j}}(\ell)=\sum_{i=1}^{M} S_{k, i \mid H_{j}} \delta\left(\ell-L_{k, i}\right)
$$


with $S_{k, i \mid H_{j}}=\int_{\Re_{k},} f_{z_{k} \mid H_{j}}(z) d z$ and $\delta(\bullet)$ is the Dirac delta function. Substituting (11) into (10) leads to

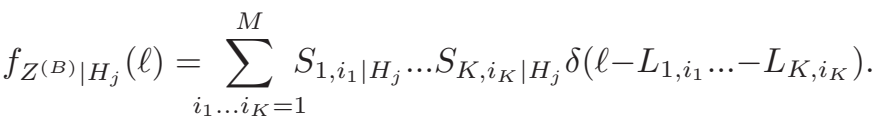

So $f_{Z(B) \mid H_{j}}$ is a pmf, where the $q$-th level is denoted $L_{q}$. Thus,

$$
f_{Z^{(B)} \mid H_{j}}(\ell)=\sum_{q} \psi_{q \mid H_{j}} \delta\left(\ell-L_{q}\right)
$$

where $\psi_{q \mid h_{j}}$ is the probability of the level $L_{q}$ and is given by $\psi_{q \mid h_{j}}=\sum_{i_{1} \ldots i_{K} \in \mathcal{L}_{q}} S_{1, i_{1} \mid H_{j}} \ldots S_{K, i_{K} \mid H_{j}}$ with $\mathcal{L}_{q}=$ $\left\{i_{1} \ldots i_{K} \mid L_{1, i_{1}}+\ldots+L_{K, i_{K}}=L_{q}\right\}$. The algorithms for computing $\psi_{q \mid H_{j}}$ and $L_{q}$ are presented in [18].

Given the pmf of $Z^{(B)}$, the false-alarm and the detection probabilities of the test can be expressed by

$$
\begin{aligned}
& P_{F}\left(B, \eta^{(B)}\right)=\sum_{q \mid L_{q} \geq \eta^{(B)}} \psi_{q \mid H_{0}}, \\
& P_{D}\left(B, \eta^{(B)}\right)=\sum_{q \mid L_{q} \geq \eta^{(B)}} \psi_{q \mid H_{1}} .
\end{aligned}
$$

\section{OPTIMAL QUANTIZED COOPERATIVE SENSING}

According to [6], the normalized throughput of a CR network is approximately given by

$$
R=\frac{T-T_{S}-T_{R}}{T} \pi_{0} C_{0}\left(1-P_{F}\right)
$$

where $C_{0}$ is the data rate per channel used for secondary user when primary user is absent. As shown in Fig. 1, a time frame length $T$ is divided into the sensing time $T_{S}$, the reporting time $T_{R}$ and the data time $T_{D}\left(T_{D}=T-T_{S}-T_{R}\right)$. Let $f_{R}$ the bandwidth devoted to the reporting channel, then $T_{R}=$ $K B / f_{R}$. The normalized throughput becomes

$$
R(N, B, \eta) \propto\left(1-\frac{N}{T f_{S}}-\frac{K B}{T f_{R}}\right)\left(1-P_{F}\right) .
$$

For the CR network with $K$ users, the throughput for the secondary user strongly depends on the cooperative sensing process, especially on the following parameters: the number of sensing samples, the number of reported bits and the optimal threshold of the global test. Therefore, optimizing these parameters to maximize the network throughput for a target detection probability $P_{D}^{(0)}$ is necessary. This optimization is then formulated as

$$
\left[N_{*}, B_{*}, \eta_{*}\right]=\arg \max _{N, B, \eta} R(N, B, \eta) \text {, s.t. } P_{D} \geq P_{D}^{(0)} \text {. }
$$

For a certain integer value of $N$, the number of reported bits $B$, which is also an integer, is necessarily less than $B_{\max }$ with $B_{\max }=\left\lfloor\left(T-N / f_{S}\right) f_{R} / K\right\rfloor$. In addition, $N<N_{\max }$ with $N_{\max }=T f_{S}$. Thus, the optimal solution can be obtained by a discrete search along with both $N$ and $B$. Therefore, for a given pair of $\{N, B\}$, the optimization in (15) leads to

$$
\eta_{*}^{(N, B)}=\arg \min _{\eta^{(N, B)}} P_{F}\left(N, B, \eta^{(N, B)}\right) \text { s.t. } P_{D}>P_{D}^{(0)} .
$$

Thanks to Eq. (13), it is equivalent to

$$
\begin{array}{r}
\eta_{*}^{(N, B)}=\quad \\
\quad \arg \min _{\eta^{(N, B)}} \sum_{q \mid L_{q} \geq \eta^{(N, B)}} \psi_{q \mid H_{0}} \\
\text { s.t. } \sum_{q \mid L_{q} \geq \eta^{(N, B)}} \psi_{q \mid H_{1}} \geq P_{D}^{(0)} .
\end{array}
$$

Since the sums in Eqs. (17a) and (17b) decrease with respect to $\eta^{(N, B)}$, the optimal $\eta_{*}^{(N, B)}$ is equal to the maximum level $L_{q}$ satisfying Eq. (17b). Consequently, the algorithm for finding $\left\{N_{*}, B_{*}, \eta_{*}\right\}$ is given as Algorithm 1 .

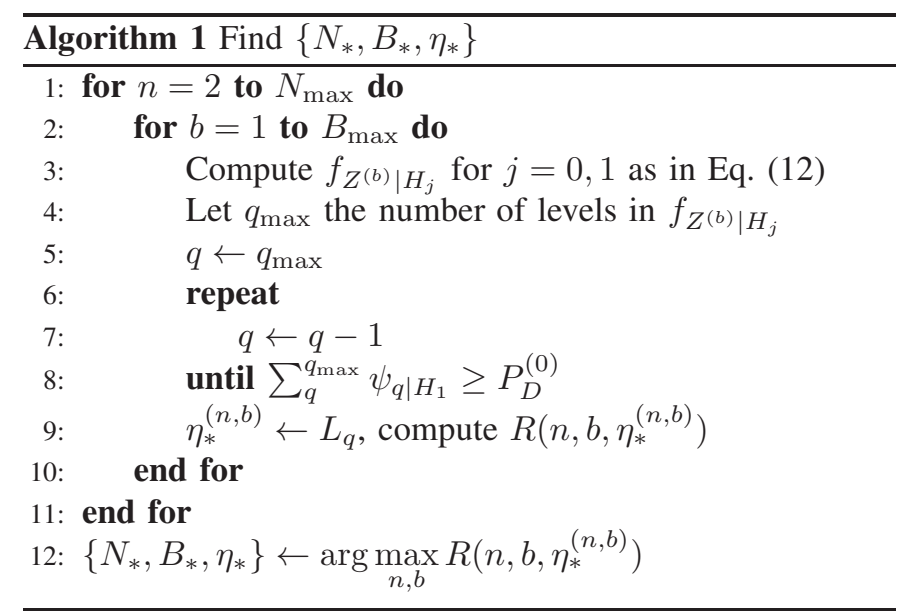

The proposed algorithm should run only when channel statistics (actually the average received SNRs at local users) have changed. Algorithm 1, including the computation of $f_{Z^{(B)} \mid H_{j}}$, is performed at the FC. The preliminary parameters for the computation of $f_{Z^{(B)} \mid H_{j}}$, i.e., the thresholds $\left\{t_{k, i}\right\}_{i=0}^{M}$, the levels $\left\{L_{k, j}\right\}_{j=1}^{M}$ and the mass coefficients $\left\{S_{k, j}\right\}_{j=1}^{M}$, can be either computed at local users and then sent to the FC, or directly computed at the FC after having received the average SNR from the local users. In both cases, the FC finally sends the optimized quantizer's configuration back to each local user.

Our work is valid for Rayleigh fading and energy detector. The extension for other fading channels is straightforward if $f_{z_{k} \mid H_{j}}$ is available in closed-form (e.g. energy detector along with a Nakagami channel [19]). When $f_{z_{k} \mid H_{j}}$ cannot be derived readily, the proposed algorithm can be adopted if the quantized version $f_{z_{k}^{(B)} \mid H_{j}}$ is achievable, e.g., based on numerical or empirical method, and stored in a lookup table.

\section{NUMERICAL RESULTS}

Unless otherwise stated, the CR network has 6 nodes and the average SNR values are $-20,-18,-16,-14,-12$, and -10 $\mathrm{dB}$, the target probability of detection $P_{D}^{(0)}$ is 0.9 , the frame length $T$ is $1 \mathrm{~ms}$, the sampling frequency $f_{S}$ is $6 \mathrm{MHz}$, and the reporting channel bandwidth $f_{R}$ is $100 \mathrm{kHz}$. The variance of the Rayleigh channel is chosen according to the SNR value.

In Fig. 2, we plot the normalized throughput versus $B$ for different SNR configurations and $N=500$. The normalized throughputs for all considered scenarios and quantization methods have the same shape and exhibit a maximum. When the number of reported bits is too small or too high, the throughput is low, due to the weak accuracy of the sensing or to the increase of the reporting time, respectively. We can see that the gaps between the maximum throughput points of the three quantizers are small, and the optimal numbers of reported bits for the three quantizers are close to each other.

In Fig. 3, we display the normalized throughput versus $N$ and $B$, when ME quantizer method is employed. The 


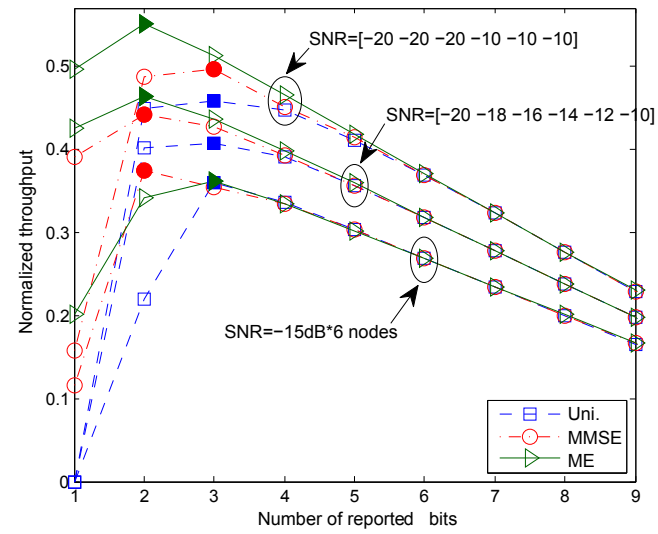

Fig. 2. Normalized throughput versus $B$.

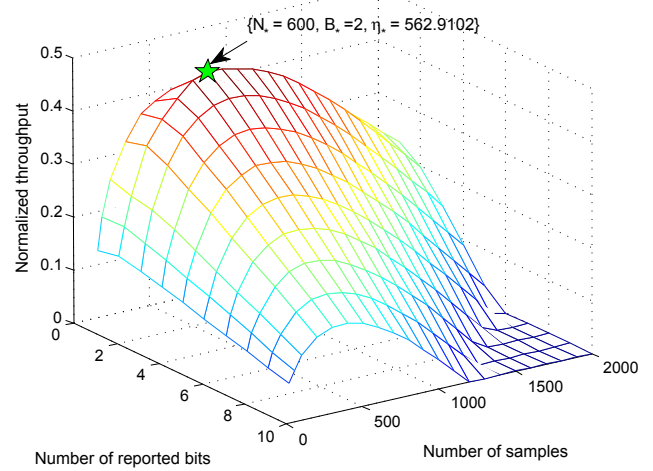

Fig. 3. Normalized throughput versus $N$ and $B$ with ME quantizer.

best combination is $N_{*}=600$ and $B_{*}=2$, which means that $10 \%$ and $2 \%$ (resp. $12 \%$ ) of the frame are devoted to sensing and reporting for each node (resp. for 6 nodes), respectively. Similar optimal combination can be obtained with other quantization methods.
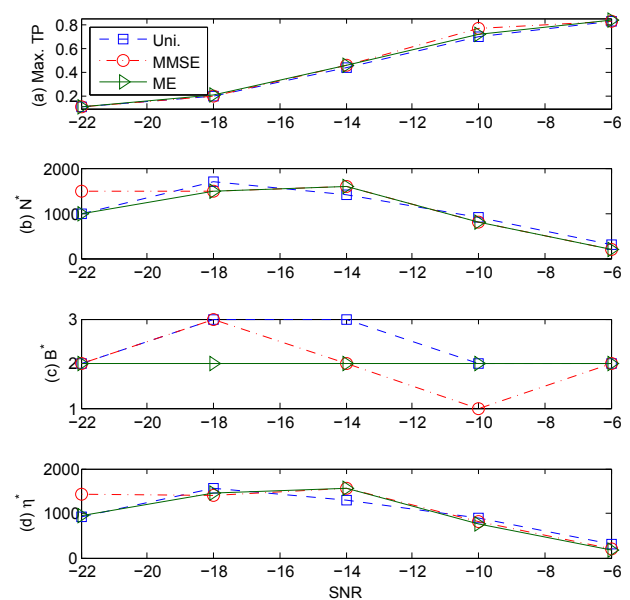

Fig. 4. Maximum normalized throughput, $N_{*}, B_{*}$ and $\eta_{*}$ versus SNR.

In Fig. 4, we plot (a) the maximum normalized throughput, (b) $N_{*}$, (c) $B_{*}$, and (d) $\eta_{*}$ versus SNR (assuming 6 nodes have identical SNRs). The throughput performance increases with
SNR. The sensing time and hence the optimal global threshold depend more strongly on the SNR than on the reporting time.

\section{CONCLUSION}

We maximized the throughput subject to a target detection probability with respect to the number of sensing samples and the number of reported bits. The proposed algorithm provides the method for selecting these parameters optimally. Reporting only a few bits is in general optimal.

\section{ACKNOWLEDGMENT}

The research leading to these results has received funding from the EC's FP7/2007-2013 under Marie Curie Fellowship CORPA, from French industry ministry in Catrene CORTIF project, and from Luxembourgish national funding FNR SATSENT.

\section{REFERENCES}

[1] E. Axell, G. Leus, E. Larsson, and H. Poor, "Spectrum sensing for cognitive radio : State-of-the-art and recent advances," IEEE Signal Processing Magazine, vol. 29, no. 3, pp. 101-116, May 2012.

[2] V. Goncalves and S. Pollin, "The value of sensing for tv white spaces," in IEEE Symposium on Dynamic Spectrum Access Networks, May 2011.

[3] S. Maleki, S. Chepuri, and G. Leus, "Optimization of hard fusion based spectrum sensing for energy-constrained cognitive radio networks," Physical Communication, vol. 9, no. 0, pp. 193 - 198, Mar 2013.

[4] N. Nguyen-Thanh and I. Koo, "Log-likelihood ratio optimal quantizer for cooperative spectrum sensing in cognitive radio," IEEE Communications Letters, vol. 15, no. 3, pp. 317-319, Mar 2011.

[5] S. Chaudhari, J. Lunden, V. Koivunen, and H. Poor, "Cooperative sensing with imperfect reporting channels: Hard decisions or soft decisions?" IEEE Trans. on Signal Processing, vol. 60, no. 1, pp. 18-28, Jan 2012.

[6] Y.-C. Liang, Y. Zeng, E. Peh, and A.-T. Hoang, "Sensing-throughput tradeoff for cognitive radio networks," IEEE Trans. on Wireless Communications, vol. 7, no. 4, pp. 1326-1337, Apr 2008.

[7] W.-Y. Lee and I. Akyildiz, "Optimal spectrum sensing framework for cognitive radio networks," IEEE Trans. on Wireless Communications, vol. 7, no. 10 , pp. $3845-3857$, Oct 2008.

[8] S. Althunibat, R. Palacios, and F. Granelli, "Performance optimization of soft and hard spectrum sensing schemes in cognitive radio," IEEE Communications Letters, vol. 16, no. 7, pp. 998-1001, Jul 2012.

[9] C. Sun, W. Zhang, and K. Ben Letaief, "Cooperative spectrum sensing for cognitive radio under bandwidth constraints," in IEEE Wireless Communications and Networking Conference, May 2007.

[10] H. Yilmaz, T. Tugcu, and F. Alagoz, "Novel quantization-based spectrum sensing scheme under imperfect reporting channel and false reports," International Journal of Communications Systems, vol. 27, no. 10, pp. 1459-1475, Oct 2014.

[11] A. Ghasemi and E. Sousa, "Opportunistic spectrum access in fading channels through collaborative sensing," Journal of Communications, vol. 2, no. 2, pp. 71-82, Feb 2007.

[12] F. Digham, M.-S. Alouini, and M. Simon, "On the energy detection of unknown signals over fading channels," IEEE Trans. on Communications, vol. 55, no. 1, pp. 21-24, Jan 2007.

[13] D. Cabric, S. Mishra, and R. Brodersen, "Implementation issues in spectrum sensing for cognitive radios," in Asilomar Conference on Signals, Systems and Computers, Nov 2004.

[14] H. Urkowitz, "Energy detection of unknown deterministic signals," Proceedings of the IEEE, vol. 55, no. 4, pp. 523-531, Apr 1967.

[15] I. Gradshteyn and I. Ryzhik, Table of Integrals, Series, and Products. Academic Press, 2007.

[16] J. Max, "Quantizing for minimum distortion," IRE Trans. on Information Theory, vol. 6, no. 1, pp. 7-12, Mar 1960.

[17] D. Messerschmitt, "Quantizing for maximum output entropy," IEEE Trans. on Information Theory, vol. 17, no. 5, p. 612, Sep 1971.

[18] D. Evans and L. Leemis, "Algorithms for computing the distributions of sums of discrete random variables," Mathematical and Computer Modelling, vol. 40, no. 13, pp. 1429 - 1452, Dec 2004.

[19] N. Reisi, M. Ahmadia, and S. Salari, "Performance analysis of energy detection-based spectrum sensing over fading," in Wireless Communication Networking and Mobile Computing, Sep 2010. 